\title{
Simultaneous Triggered Collapse of the Presolar Dense Cloud Core and Injection of Short-Lived Radioisotopes by a Supernova Shock Wave
}

\author{
Alan P. Boss ${ }^{1}$, Sergei I. Ipatov ${ }^{1}$, Sandra A. Keiser ${ }^{1}$, Elizabeth A. Myhill ${ }^{1,2}$, and Harri A. T. \\ Vanhala $^{1,3}$
}

\begin{abstract}
Cosmochemical evidence for the existence of short-lived radioisotopes (SLRI) such as ${ }^{26} \mathrm{Al}$ and ${ }^{60} \mathrm{Fe}$ at the time of the formation of primitive meteorites requires that these isotopes were synthesized in a massive star and then incorporated into chondrites within $\sim 10^{6} \mathrm{yr}$. A supernova shock wave has long been hypothesized to have transported the SLRI to the presolar dense cloud core, triggered cloud collapse, and injected the isotopes. Previous numerical calculations have shown that this scenario is plausible when the shock wave and dense cloud core are assumed to be isothermal at $\sim 10 \mathrm{~K}$, but not when compressional heating to $\sim$ $1000 \mathrm{~K}$ is assumed. We show here for the first time that when calculated with the FLASH2.5 adaptive mesh refinement (AMR) hydrodynamics code, a $20 \mathrm{~km} / \mathrm{sec}$ shock wave can indeed trigger the collapse of a $1 M_{\odot}$ cloud while simultaneously injecting shock wave isotopes into the collapsing cloud, provided that cooling by molecular species such as $\mathrm{H}_{2} \mathrm{O}, \mathrm{CO}_{2}$, and $\mathrm{H}_{2}$ is included. These calculations imply that the supernova trigger hypothesis is the most likely mechanism for delivering the SLRI present during the formation of the solar system.
\end{abstract}

Subject headings: hydrodynamics - instabilities - solar system: formation - stars: formation

\section{Introduction}

The discovery of evidence for live ${ }^{26} \mathrm{Al}$ (half-life of $0.7 \times 10^{6} \mathrm{yr}$ ) at the time of the formation of refractory inclusions in the Allende meteorite (Lee et al. 1976) led quickly

\footnotetext{
${ }^{1}$ Department of Terrestrial Magnetism, Carnegie Institution of Washington, 5241 Broad Branch Road, NW, Washington, DC 20015-1305; boss@dtm.ciw.edu, siipatov@hotmail.com, keiser@dtm.ciw.edu.

${ }^{2}$ Marymount University, Arlington, VA 22207; elizabeth.myhill@marymount.edu.

${ }^{3}$ Universities Space Research Association, Columbia, MD 21044; hvanhala@usra.edu.
} 
to the suggestion that the ${ }^{26} \mathrm{Al}$ was synthesized in a supernova, then transported by the supernova's shock wave to a dense molecular cloud, where the shock wave triggered the collapse of a cloud core and injected the ${ }^{26} \mathrm{Al}$ into the collapsing presolar cloud (Cameron and Truran 1977). Detailed numerical calculations showed that such simultaneous triggering and injection was possible, provided that the shock wave had slowed to speeds of $\sim 10$ to $~$ $40 \mathrm{~km} / \mathrm{sec}$ (Boss 1995; Foster \& Boss 1996, 1997) and that the shock wave and cloud were effectively isothermal at $\sim 10 \mathrm{~K}$. This isothermal assumption is appropriate for the radiative phase of a supernova shock (Chevalier 1974), which occurs when the shock has travelled $10 \mathrm{pc}$ and swept up a cool shell of gas and dust. Recent adaptive mesh refinement (AMR) studies (Nakamura et al. 2006; Melioli et al. 2006) have shown that shock-triggered star formation is likely to occur when the supernova shock has evolved into a radiative shock, i.e., the shock wave is able to cool so rapidly by radiation that the shock wave is effectively at the same temperature as the ambient medium, confirming the results of Boss (1995) and Foster \& Boss $(1996,1997)$.

Vanhala \& Cameron (1998; hereafter VC) used a smoothed particle hydrodynamics (SPH) code and detailed equations of state to confirm the previous results for isothermal shocks and clouds. However, when VC allowed their models to become nonisothermal by including compressional heating and molecular, atomic, and dust cooling, they found that they could not achieve simultaneous triggering of cloud collapse and injection of shock wave material, raising serious doubts about the supernova trigger hypothesis.

Alternative explanations for the origin of the SLRI in chondritic meteorites have also arisen. Shu et al. (1997) suggested that the SLRI were produced in situ by spallation reactions involving energetic particles emanating from protosolar flares striking dust grains near the $\mathrm{X}$-wind point, from whence the grains would be lofted upward by the X-wind and transported outward to the solar nebula. However, the SLRI ${ }^{60} \mathrm{Fe}$ cannot be produced by spallation, and requires a stellar nucleosynthetic source (Tachibana \& Huss 2003) such as a supernova. The fact that the results of ${ }^{26} \mathrm{Al}$ dating agree well with the independent $\mathrm{Pb}-\mathrm{Pb}$ dating system (Connelly et al. 2008) implies that the ${ }^{26} \mathrm{Al}$ was spatially homogeneous in the solar nebula, which is inconsistent with the bulk of its production by spallation reactions at the X-wind point. Regardless of whether spallation reactions contributed to the SLRI inventory, then, a supernova source for the ${ }^{26} \mathrm{Al}$ and ${ }^{60} \mathrm{Fe}$ appears to be necessary.

Given a supernova origin for the ${ }^{26} \mathrm{Al}$ and ${ }^{60} \mathrm{Fe}$, it has also been suggested that a nearby $(\sim 0.1 \mathrm{pc})$ supernova may have injected these SLRI into the already-formed solar nebula (Ouellette, Desch \& Hester 2007). While such hot shock front gas is unable to penetrate into the much denser disk gas (Chevalier 2000), Ouellette et al. (2007) suggested that SLRI residing in dust grains micron-sized and larger shot through the stalled shock-wave gas and 
into the disk. However, supernova dust grains are essentially all smaller than 0.1 micron, and are sputtered to even smaller sizes in the shock (Bianchi \& Schneider 2007). Gounelle \& Meibom (2008) noted that disks formed within $\sim 0.3$ pc of a massive star would be photoevaporated away prior to its supernova explosion. Furthermore, Krot et al. (2008) argued that injection into a late-phase solar nebula would have led to the injection of stable oxygen isotopes as well as SLRI into the disk, leading to an oxygen isotope distribution that would be inconsistent with the observed values and their explanation by mass-independent fractionation. Krot et al. (2008) and Thrane et al. (2008) argued that injection must have occurred instead into the presolar cloud, so that the sun and the solar nebula shared a common reservoir of oxygen isotopes.

Here we return to the question of whether nonisothermal shock fronts are fatal for the supernova triggering hypothesis, by using the FLASH2.5 AMR code and an improved cooling law to reinvestigate this basic question of solar system origin.

\section{Numerical Methods and Initial Conditions}

FLASH employs a block-structured adaptive grid approach. Advection is handled by the piecewise parabolic method (PPM), featuring a Riemann solver at cell boundaries that handles shock fronts exceptionally well. We downloaded and ran the FLASH2.5 AMR code (flash.uchicago.edu/website/home) on several of the FLASH-supplied test problems relevant to shock-triggered star formation, namely the Sod shock tube problem and the gravitational collapse of a pressureless cloud. We then used FLASH2.5 to reproduce the standard case of triggered collapse of Foster \& Boss (1996), and verified that FLASH2.5 was able to produce simultaneous triggered collapse and injection of shock wave material when the shocked cloud was forced to remain isothermal at $10 \mathrm{~K}$. The details of these test cases will be presented in a future paper.

In the present models, we used the two dimensional, cylindrical coordinate $(R, Z)$ version of FLASH2.5, with axisymmetry about the rotational axis $(\hat{z})$. Multipole gravity was used, including Legendre polynomials up to $l=10$. The equation of state routines were taken to be those for a simple perfect gas with a mean molecular weight of $\mu=2.3$.

As in Foster \& Boss (1996), the target dense cloud core is a stable Bonnor-Ebert (BE) sphere with a mass of $1 M_{\odot}$, a radius of $0.058 \mathrm{pc}$, a temperature of $T=10 \mathrm{~K}$, and a maximum density of $6.2 \times 10^{-19} \mathrm{~g} \mathrm{~cm}^{-3}$ at rest near the top of the cylindrical grid. The BE sphere is

embedded in an intercloud medium with a density of $3.6 \times 10^{-22} \mathrm{~g} \mathrm{~cm}^{-3}$ and a temperature of $10 \mathrm{~K}$. The shock wave begins at the top of the grid and propagates downward at $20 \mathrm{~km} / \mathrm{sec}$ 
toward the BE sphere. The shock wave has a thickness of $0.003 \mathrm{pc}$ with a uniform density of $3.6 \times 10^{-20} \mathrm{~g} \mathrm{~cm}^{-3}$ and a temperature of $1000 \mathrm{~K}$. The shock wave is followed by a wind with a density of $3.6 \times 10^{-22} \mathrm{~g} \mathrm{~cm}^{-3}$ and temperature of $1000 \mathrm{~K}$ also moving downward at $20 \mathrm{~km} / \mathrm{sec}$. The shock wave material is represented by a color field, initially defined to be equal to 1 inside the shock wave and 0 elsewhere, which allows the shock wave material to be tracked in time (Foster \& Boss 1997). The SLRI are assumed to be contained in dust grains of sub-micron size (Bianchi \& Schneider 2007), small enough for the grains to remain coupled to the gas. These initial conditions are identical to those in the standard case of Foster \& Boss (1996), with the exception of the temperatures of $1000 \mathrm{~K}$ in the shock and wind, and the nonzero velocity of the wind.

The cylindrical grid is $0.197 \mathrm{pc}$ long in $Z$ and $0.063 \mathrm{pc}$ wide in $R$. We set the number of blocks in $R$ to be 5 and in $Z$ to be 15 , leading to approximately uniform spacing in $R$ and $Z$, with each block consisting of $8 \times 8$ grid points, equivalent to an initial grid of $40 \times 120$. With up to five levels of refinement allowed, FLASH is then able to follow smallscale structures with the effective resolution of a grid 16 times finer in scale, or effectively $640 \times 1920$, somewhat higher than the highest resolution of $480 \times 1440$ used by Vanhala \& Boss (2000), but less than the resolution of $960 \times 2880$ used by Vanhala \& Boss (2002).

\section{Results}

In the absence of cooling, FLASH produces an adiabatic evolution with an effective $\gamma=5 / 3$. Model NC was run without cooling, but with the temperature constrained to be less than $1000 \mathrm{~K}$. The shock wave was unable to compress any part of the target cloud to a density higher than about twice that of the initial central density of the cloud. Much of the cloud was heated to temperatures of $100 \mathrm{~K}$ to $1000 \mathrm{~K}$, preventing cloud collapse. Instead, the cloud's remnants were swept up by the shock wave and wind and transported off the grid. This result is quite similar to that obtained by Foster \& Boss (1996) for the standard case when run with an adiabatic pressure law $(\gamma=5 / 3)$ rather than an isothermal pressure law.

We next ran three models with variations in the cooling function, based on the results of Neufeld \& Kaufman (1993), who calculated the radiative cooling caused by rotational and vibrational transitions of optically thin, warm molecular gas composed of $\mathrm{H}_{2} \mathrm{O}, \mathrm{CO}$, and $\mathrm{H}_{2}$, finding $\mathrm{H}_{2} \mathrm{O}$ to be the dominant cooling agent. Their Figure 3 shows that over the range of temperature from $100 \mathrm{~K}$ to $4000 \mathrm{~K}$, the total cooling rate coefficient $L$ can be approximated as $L \approx L_{0} \approx 10^{-24}(T / 100) \mathrm{erg} \mathrm{cm}^{3} \mathrm{~s}^{-1}$. The cooling rate $\Lambda=L n\left(H_{2}\right) n(m)$, where $n\left(H_{2}\right)$ is the number density of molecular hydrogen and $n(m)$ is the number density 
of the molecular species under consideration. Assuming that $n\left(\mathrm{H}_{2} \mathrm{O}\right) / n\left(\mathrm{H}_{2}\right) \approx 8.8 \times 10^{-4}$, we take $n(m) / n\left(H_{2}\right) \approx 10^{-3}$, leading to $\Lambda \approx 9 \times 10^{19}(T / 100) \rho^{2} \mathrm{erg} \mathrm{cm}^{-3} \mathrm{~s}^{-1}$, where $\rho$ is the gas density in $\mathrm{g} \mathrm{cm}^{-3}$.

The figures show the results of model C, which used the Neufeld \& Kaufman (1993) cooling rate as well as the constraint that the temperatures remain at $1000 \mathrm{~K}$ or less. Figure 1 shows that Rayleigh-Taylor (R-T) fingers form immediately after the shock strikes the cloud and penetrate downward farthest along the symmetry axis. Kelvin-Helmholtz (K-H) rolls form soon thereafter, as the shock front ablates material off the sides of the cloud and transports it downstream. The color field is injected into the target cloud by a combination of the R-T and K-H instabilities, though the cloud material polluted by the $\mathrm{K}-\mathrm{H}$ vortices tends to be lost by subsequent ablation; the R-T fingers have the best chance to inject shock wave material close to the cloud's symmetry axis and hence into the region where a protostar will soon form. The fact that both gas and dust from the shock wave region are directly injected into the cloud by R-T fingers ensures that SLRI carried by the shock wave will also be injected, as found in the previous isothermal models (Foster \& Boss 1997; Vanhala \& Boss 2000, 2002)

Figure 2 shows that after $0.1 \mathrm{Myr}$, a region along the symmetry axis has formed with a maximum density $\sim 1000$ times that of the center of the initial target cloud. The temperature contours show that the thermal energy generated by compressional heating at the shock-cloud interface is rapidly lost by the molecular cooling. The maximum temperatures of $1000 \mathrm{~K}$ are limited to thin shells at the shock-cloud interface; cooling is so rapid in the denser regions just inside this interface that the temperature falls to $10 \mathrm{~K}$, the minimum temperature allowed by the calculation. This rapid cooling makes the evolution similar to that of isothermal models.

Figures 3 and 4 show a close-up of the forming protostar along the symmetry axis, after $0.16 \mathrm{Myr}$, when the maximum density has reached $2 \times 10^{-12} \mathrm{~g} \mathrm{~cm}^{-3}$. Lower density regions continue to infall onto the growing central protostar with velocities on the order of 1 $\mathrm{km} / \mathrm{sec}$, highly supersonic compared to the sound speed for $10 \mathrm{~K}$ gas of $0.2 \mathrm{~km} / \mathrm{sec}$. Clearly the protostar has entered the dynamic collapse phase, and will form a central protostellar core surrounded by an infalling envelope. Figure 4 shows that while the protostar is polluted with shock wave material, the lower density gas that will soon accrete onto the protostar typically has an even higher density of shock wave material than that already in the protostar. Note that the present models do not include rotation of the target cloud, so a protoplanetary disk cannot form in these models.

We estimate the injection efficiency of the shock wave material into the forming protostar by calculating the amount of color residing inside regions with density greater than $10^{-18}$ $\mathrm{g} \mathrm{cm}^{-3}$ at the time $(0.16 \mathrm{Myr})$ shown in Figures 3 and 4 . The fraction of the incident 
color field that was injected into this infalling region is $\sim 0.003$ for model $\mathrm{C}$. This low injection efficiency is similar to that $(\sim 0.002)$ obtained by Vanhala \& Boss $(2002)$ in their extremely high resolution, isothermal shock-cloud models, for a comparable definition of injection efficiency.

Such a low injection efficiency is in accord with a supernova as the source of the shock wave. Based on the estimates of Cameron et al. (1995), Foster \& Boss (1997) noted that the ${ }^{26} \mathrm{Al}$-containing gas and dust in a supernova shock wave would have to be diluted by a factor of $\sim 10^{4}$ in order to explain the inferred initial abundance of ${ }^{26} \mathrm{Al}$ in the solar nebula, i.e., $10^{-4} M_{\odot}$ of shock-wave material should be injected into a $1 M_{\odot}$ presolar cloud. In the standard case, the mass of the shock wave that is incident on the target cloud is $0.016 M_{\odot}$, so the desired injection efficiency is $\sim 0.006$. However, given that not all of the $1 M_{\odot}$ target cloud will be accreted by the protostar, the injection efficiency would need to be half as large if only $0.5 M_{\odot}$ is accreted, dropping the desired injection efficiency to $\sim 0.003$, the same as that obtained in model C. Many other factors enter into the desired injection efficiency, such as radioactive decay, nucleosynthetic yields (Rauscher et al. 2002), and sweeping-up of intervening interstellar cloud material, so these estimates should only be taken as being consistent to order of magnitude.

Two other models with cooling were calculated, identical to model $\mathrm{C}$ except for having the Neufeld \& Kaufman (1993) cooling rate doubled (model 2C) or halved (model 0.5C), in order to test the sensitivity of the results to the assumed cooling rate. Both models evolved very similarly to model $\mathrm{C}$, with the main difference being that the amount of injected shock front material was about $1 / 3$ higher in model $2 \mathrm{C}$ and about $1 / 10$ lower in model $0.5 \mathrm{C}$. Evidently simultaneous triggering and injection is relatively insensitive to changes in $\Lambda$ of this magnitude.

Finally, it is worth considering why these results differ from those of VC, who were unable to achieve simultaneous triggering and injection in their three dimensional SPH models with cooling. The reasons appear to be three-fold. First, the FLASH AMR code with PPM is superb at representing the physics of shock waves striking target clouds, such as the R-T and $\mathrm{K}-\mathrm{H}$ instabilities that dominate these interactions. SPH has its strengths, but SPH is relatively poor at resolving the R-T and K-H dynamical instabilities (e.g., Agertz et al. 2007). Second, the Neufeld \& Kaufman (1993) cooling rate estimate is a considerable improvement over the cooling rates used in $\mathrm{VC}$, which included many species, but did not include the contribution due to $\mathrm{H}_{2} \mathrm{O}$, which Neufeld \& Kaufman (1993) found to be dominant. Third, and perhaps most importantly, low injection efficiencies would be hard to detect in the VC SPH calculations, which typically involved only 5000 particles in the target cloud, so that the desired dilution factor of $10^{4}$ for a supernova shock wave (Foster \& Boss 1997) would 
require that only half a particle be injected.

\section{Conclusions}

These models show that when cooling by molecular species is included, a $20 \mathrm{~km} / \mathrm{sec}$ shock wave is able to trigger the gravitational collapse of an otherwise stable, solar-mass dense cloud core, as well as to inject appropriate amounts of supernova shock wave material into the collapsing cloud core. This injected material consists of shock wave gas as well as dust grains small enough to remain coupled to the gas, i.e., sub-micron-sized grains, which are expected to characterize supernova shock waves (Bianchi \& Schneider 2007) and to carry the SLRI whose decay products have been found in refractory inclusions of chondritic meteorites (Lee et al. 1976). Evidently a radiative-phase supernova shock wave is able to cool sufficiently rapidly to behave in much the same way as a shock wave that is assumed to remain isothermal with the target cloud (Boss 1995; Foster \& Boss 1996, 1997; Vanhala \& Boss 2000, 2002). These models thus lend strong support to the hypothesis first advanced by Cameron \& Truran (1977) that a supernova shock wave carrying SLRI may have triggered the formation of the solar system.

We thank Tomasz Plewa for valuable assistance with using FLASH2.5 and Roger Chevalier, James Stone, and the two referees for their comments. This research was supported in part by NASA Origins of Solar Systems grant NNG05GI10G and NASA Planetary Geology and Geophysics grant NNX07AP46G, and is contributed in part to NASA Astrobiology Institute grant NCC2-1056. The software used in this work was in part developed by the DOE-supported ASC/Alliances Center for Astrophysical Thermonuclear Flashes at the University of Chicago.

\section{REFERENCES}

Agertz, O., et al. 2007, MNRAS, 380, 963

Bianchi, S., \& Schneider, R. 2007, MNRAS, 378, 973

Boss, A. P. 1995, ApJ, 439, 224

Cameron, A. G. W., \& Truran, J. W. 1977, Icarus, 30, 447

Cameron, A. G. W., Höflich, P., Myers, P. C., \& Clayton, D. D. 1995, ApJ, 447, L53

Chevalier, R. 1974, ApJ, 188, 501 
-. 2000, ApJ, 538, L151

Connelly, J. N., Amelin, Y., Krot, A. N., \& Bizzarro, M. 2008, ApJ, 675, L121

Foster, P. N., \& Boss, A. P. 1996, ApJ, 468, 784

-. 1997, ApJ, 489, 346

Gounelle, M. \& Meibom, A. 2008, ApJ, 680, 781

Krot, A. N., et al. 2008, ApJ, 672, 713

Lee, T., Papanastassiou, D. A., \& Wasserburg, G. J. 1976, Geophys. Res. Lett., 3, 109

Melioli, C., de Gouveia Dal Pino, E. M., de la Reza, R., \& Raga, A. 2006, MNRAS, 373, 811

Nakamura, F., McKee, C. F., Klein, R. I., \& Fisher, R. T. 2006, ApJS, 164, 477

Neufeld, D. A., \& Kaufman, M. J. 1993, ApJ, 418, 263

Ouellette, N., Desch, S. J., \& Hester, J. J. 2007, ApJ, 662, 1268

Rauscher, T., Heger, A., Hoffman, R. D., \& Woosley, S. E. 2002, ApJ, 576, 323

Shu, F. H., Shang, H., Glassgold, A. E., \& Lee, T. 1997, Science, 277, 1475

Tachibana, S., \& Huss, G. R. 2003, ApJ, 588, L41

Thrane, K., Nagashima, K., Krot, A. N., \& Bizzarro, M. 2008, ApJ, 680, L141

Vanhala, H. A. T., \& Boss, A. P. 2000, ApJ, 538, 911

—. 2002, ApJ, 575, 1144

Vanhala, H. A. T., \& Cameron, A. G. W. 1998, ApJ, 508, 291 (VC) 


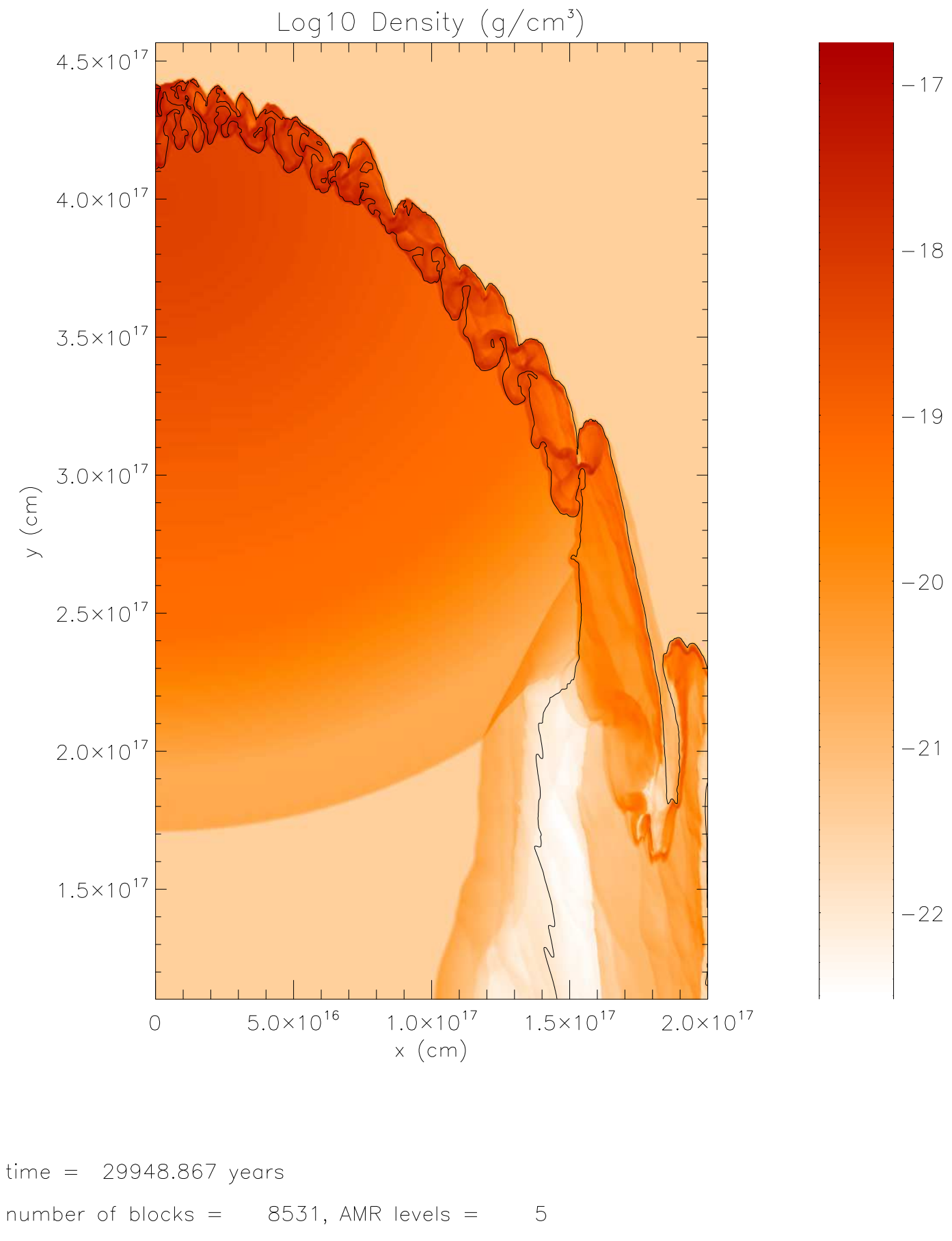

Fig. 1.- Density for model C after $0.03 \mathrm{Myr}$ of evolution. Contours show regions with color fields (representing SLRI) greater than 0.01. Symmetry axis is along the left hand side of the plot. Shock wave travels downward from top of box. R-T fingers and $\mathrm{K}-\mathrm{H}$ vortices form at the shock-cloud interface. $R$ axis is horizontal and $Z$ axis is vertical. 


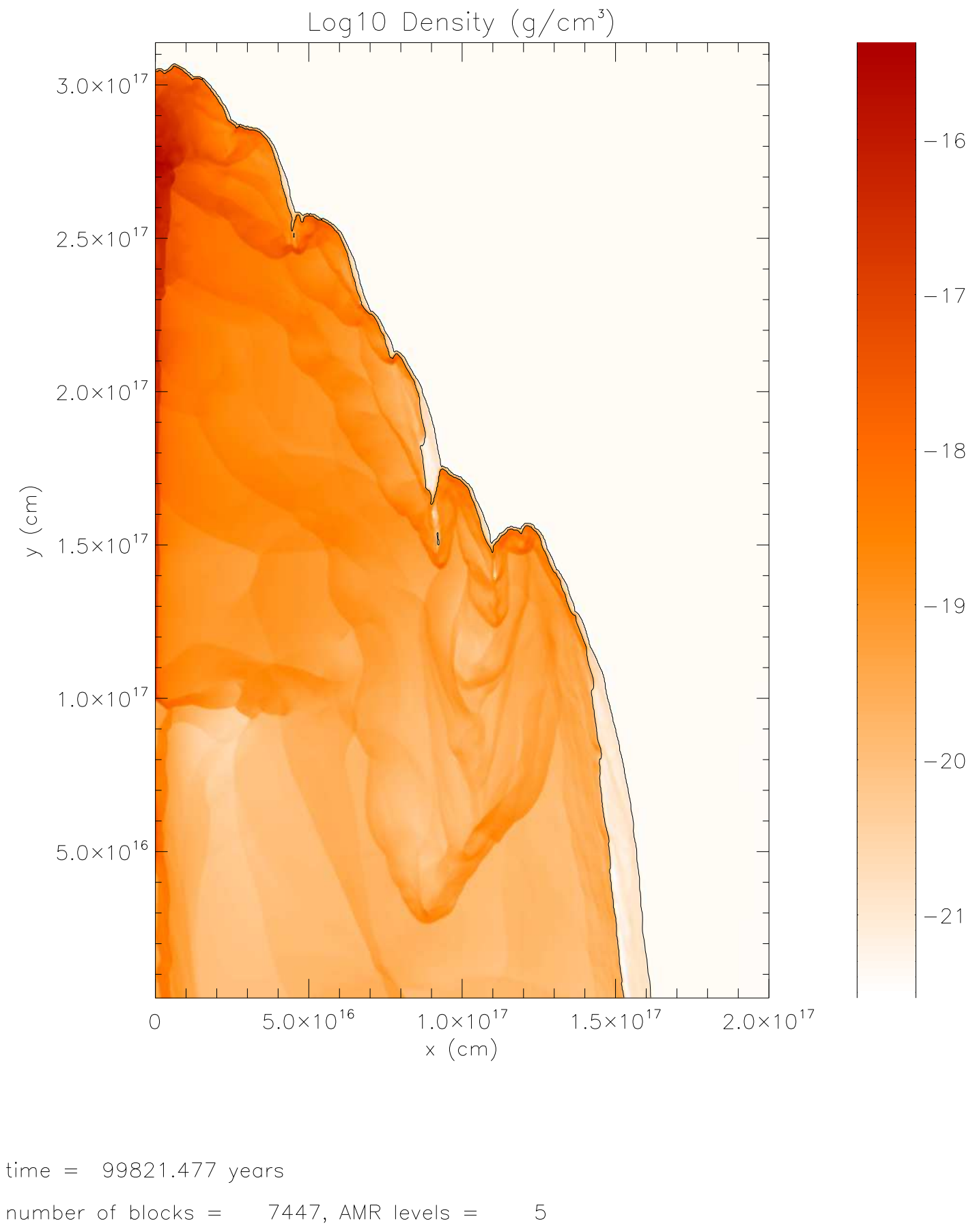

Fig. 2.- Same as Figure 1 except after 0.1 Myr. Contours now show regions with temperatures greater than $100 \mathrm{~K}$, which only occur at the shock-cloud interface as a result of the molecular cooling. A high-density region has formed along the symmetry axis. 

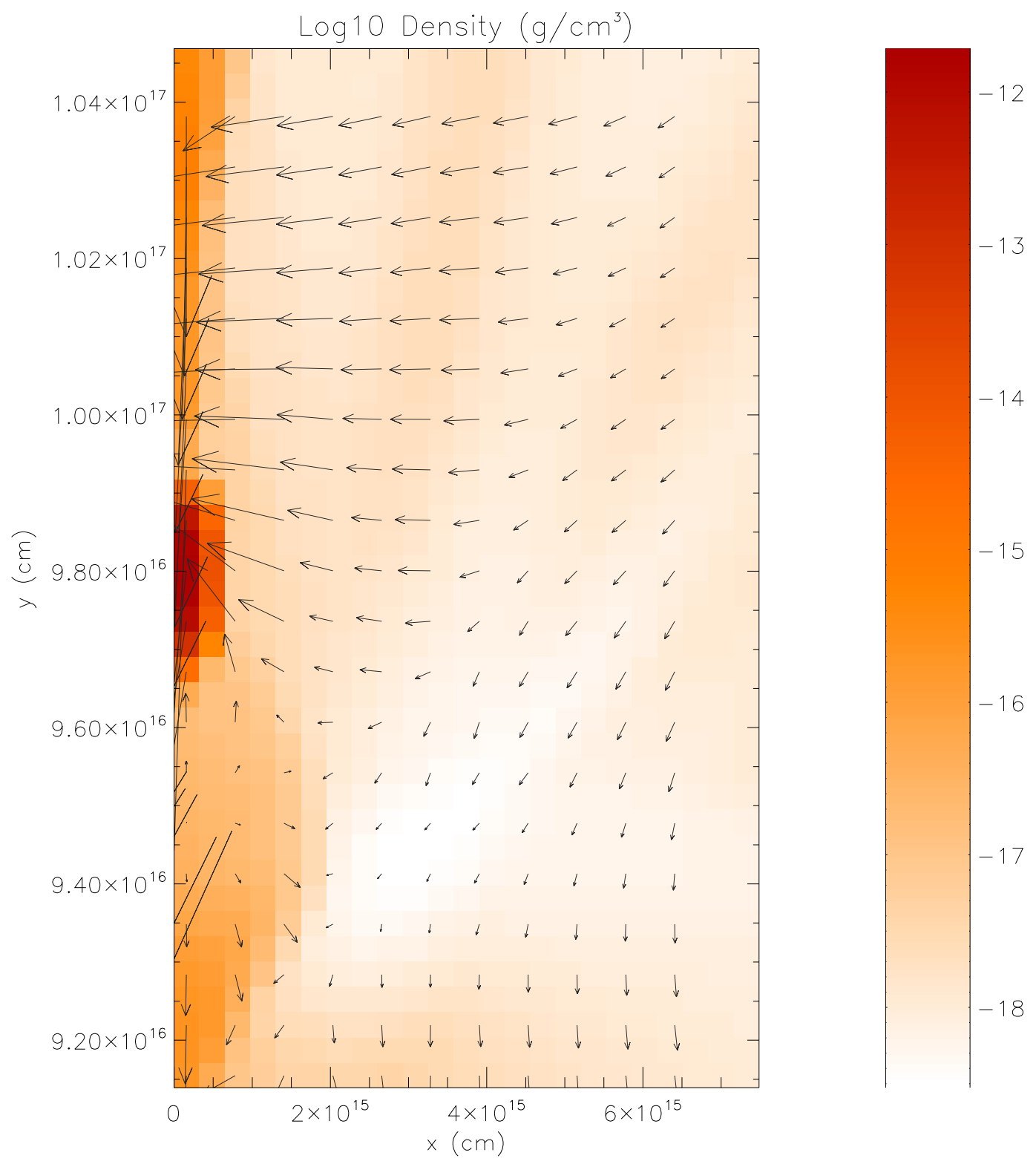

time $=159712.516$ years

number of blocks $=1691$, AMR levels $=$

5

Fig. 3.- Same as Figure 1 except after $0.16 \mathrm{Myr}$ and limited to a small region around the density maximum of $\sim 2 \times 10^{-12} \mathrm{~g} \mathrm{~cm}^{-3}$. Velocity contours are shown for every other AMR grid cell. Much of the cloud is infalling onto the growing protostar on the symmetry axis. 


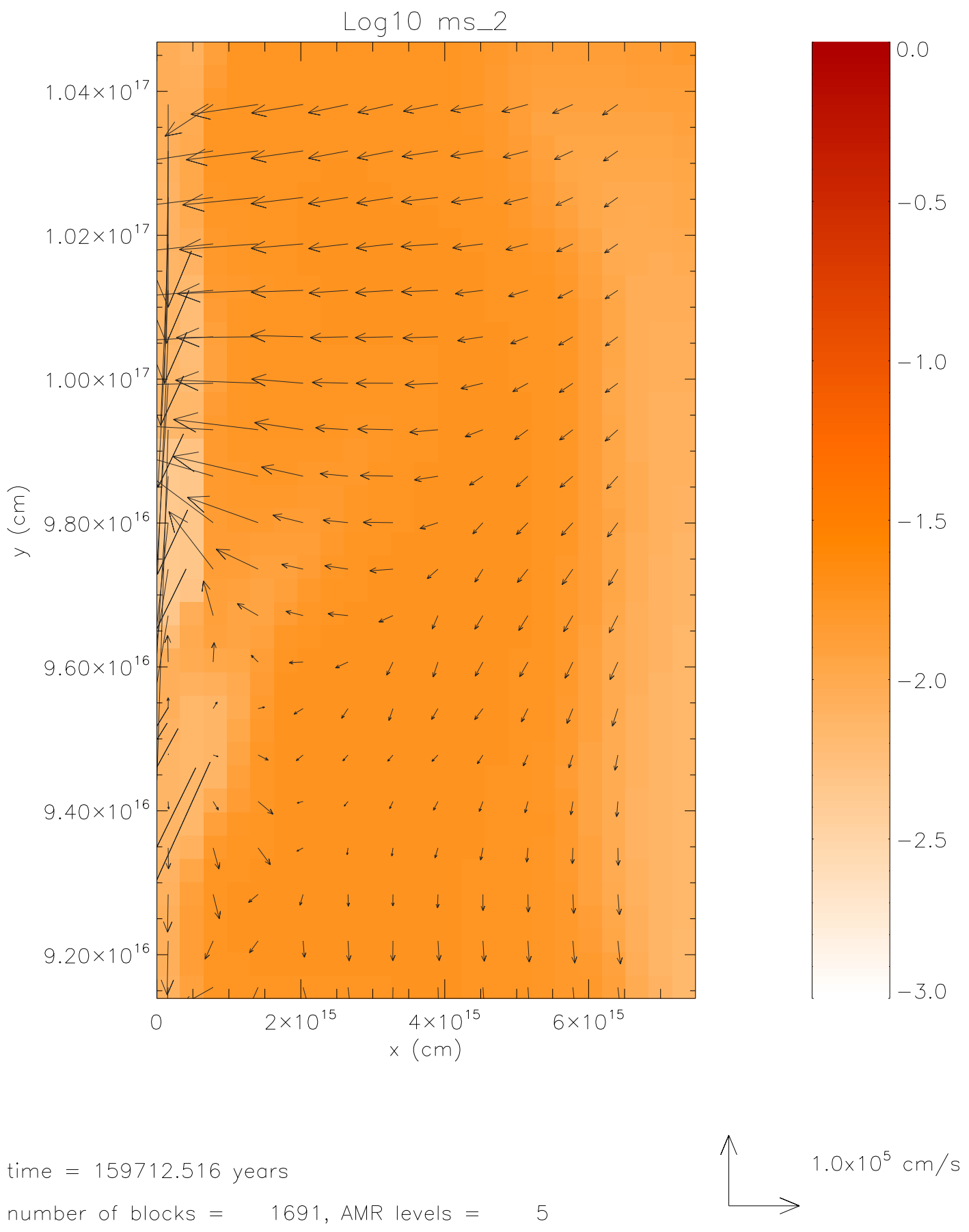

Fig. 4.- Same as Figure 3 except now the color field is plotted along with the velocity vectors, showing that while the growing protostar contains some color, the infalling regions contain a higher color density, i.e., more a higher density of SLRI from the shock wave. 